\title{
EFECTO DE LA APLICACIÓN DE ENMIENDAS LÍQUIDAS EN EL SUELO Y EN EL CRECIMIENTO DE MAÍZ BAJO CONDICIONES DE INVERNADERO ${ }^{1}$
}

\author{
Gabriel Garbanzo-León ${ }^{*}$ Eloy Molina-Rojas ${ }^{2 / *}$, Gilberto Cabalceta-Aguilar ${ }^{*}$ \\ Palabras clave: Aluminio; enmienda; calcio; ultisol; maíz. \\ Keywords: Aluminum; lime; calcium; ultisol; corn.
}

Recibido: 10/02/16

RESUMEN

Se evaluó el efecto de 3 enmiendas líquidas en la fertilidad de un Ultisol, cultivado con maíz bajo condiciones de invernadero. El estudio se realizó en macetas de 1,0 y 0,5 1 de suelo proveniente de Orosí, Cartago, con acidez intercambiable de 4,64 $\mathrm{cmol}^{-1} \mathrm{l}^{-1}$, a partir de enmiendas líquidas de Cal 56 (C56) y Surkal (SK) y el carbonato de calcio en polvo mojable Surflow Calcio (SC), diluidas en agua y aplicadas en 2 dosis cada una. Se realizaron análisis de $\mathrm{pH}$ y acidez intercambiable en diferentes momentos durante un período de 61 días después de aplicadas (dda) las enmiendas, en macetas sin plantas. También se realizaron análisis de fertilidad a los 45 días después de la siembra, y se llevaron a cabo mediciones de altura de las plantas, largo de las raíces y peso seco de la biomasa del maíz, en macetas con plantas. La aplicación de las enmiendas C56 y SK disminuyó la acidez intercambiable y elevó el $\mathrm{pH}$ en forma proporcional a la dosis durante los primeros 14 dda. A partir de los 27 días la acidez empezó a subir y el pH a bajar. Con el SC la acidez se mantuvo baja y el $\mathrm{pH}$ alto durante todo el período de evaluación debido que se aplicó una dosis mayor que las enmiendas líquidas. La fertilidad del suelo mejoró con la aplicación de enmiendas, principalmente en los tratamientos

1 Proyecto VI-733-B0-137 Manejo de la acidez del suelo y la nutrición de cultivos tropicales.

2 Autor para correspondencia. Correo electrónico: eloy.molina@ucr.ac.cr
Aceptado: 04/07/16
* Universidad de Costa Rica, Centro de investigaciones Agronómicas, Costa Rica. 
de SC. La aplicación de enmiendas incrementó la altura de las plantas, longitud de las raíces y el peso seco de la biomasa del maíz. El SK produjo mayor acumulación de materia seca aérea, sin embargo, con el producto C56 se determinó una mayor densidad longitudinal de raíces. Se concluye que las cales en presentación líquida pueden disminuir la acidez del suelo de manera rápida con un efecto residual superior a los 61 días, siendo una alternativa potencial para etapas fenológicas críticas bajo condiciones de invernadero.

\section{INTRODUCCIÓN}

La acidez es uno de los principales factores de la degradación química de los suelos en regiones tropicales (Bertsch 1995), debido principalmente a la meteorización intensiva a través del tiempo en un ambiente de alta precipitación y temperatura, condiciones que favorecen la pérdida de bases por lixiviación y lavado (Molina 1998). El origen de la acidez es un proceso espontáneo durante la pedogénesis, lo cual consiste en la pérdida de cationes $\left(\mathrm{K}^{+}, \mathrm{Na}^{+}, \mathrm{Ca}^{+2}, \mathrm{Mg}^{+2}\right)$ y un incremento de cationes metálicos $\left(\mathrm{Al}^{+3}\right.$, $\left.\mathrm{Fe}^{+3}, \mathrm{Mn}^{+4}\right)$ que sufren el efecto de hidrólisis ácida (Zapata 2004). La meteorización también favorece la pérdida de $\mathrm{Si}$ en forma de $\mathrm{Si}(\mathrm{OH})_{4}$ (Quero 2005), y junto con la hidrolisis del $\mathrm{Al}^{3+}$ intercambiable causa la formación de iones $\mathrm{H}^{+}$en el suelo, que contribuyen a disminuir el $\mathrm{pH}$ del suelo (Alvarado y Fallas 2004).

La acidez del suelo también puede ser causada por la acción directa del hombre con el uso de fertilizantes nitrogenados amoniacales que liberan iones $\mathrm{H}^{+}$a través de la nitrificación del $\mathrm{NH}_{4}{ }^{+}$(Espinosa y Molina 1999). La erosión del suelo y la lluvia ácida proveniente de la mezcla de gases de fábricas y erupciones volcánicas con el agua, también contribuyen a la acidificación del suelo (Molina 1998). application increased plant height, root length, and dry corn biomass weight. SK produced higher aerial dry matter accumulation; however, with C56 a greater longitudinal root density was determined. It is concluded that liquid lime can reduce soil acidity rapidly with more than 61 days residual effect, being a potential alternative for critical growth stages in crops under greenhouse conditions.

La acidificación del suelo disminuye el pH (Molina 1998, Espinosa y Molina 1999), lo cual repercute en la solubilidad de algunos nutrimentos. Por debajo de pH 5,5 el Al, Fe y Mn se vuelven más solubles y pueden afectar el crecimiento de las raíces de las plantas, mientras que a pH de 6,5 a 7,5 la solubilidad de los nutrimentos esenciales para las plantas es mayor (Marschner y Marschner 2012).

El manejo de la acidez se efectúa mediante el desplazamiento de los Iones de $\mathrm{Al}^{+3}$ por cationes como el $\mathrm{Ca}$ y $\mathrm{Mg}$ cuando se aplican enmiendas al suelo, que lleva a la hidrólisis del $\mathrm{Al}$ a formas monoméricas y poliméricas (hidroxi - aluminicos). $\mathrm{El} \mathrm{Al}^{+3}$ intercambiable es transformado en hidróxidos de $\mathrm{Al}$ insolubles lo que aumenta el pH del suelo (Espinosa y Molina 1999). Este efecto es constante cuando se aplican enmiendas a base de $\mathrm{CO}_{3}^{-2}, \mathrm{OH}^{-}$y $\mathrm{SIO}_{3}^{-2}$ que son responsables de la reacción neutralizante del Al (Espinosa 1995, Molina 1998).

El efecto neutralizante de las enmiendas agrícolas está basado en la determinación de la eficiencia agronómica del material, que involucra 4 factores importantes: pureza química, forma química, tamaño de partícula y Poder Relativo de Neutralización Total (PRNT) (Molina 1998). Los materiales de enmienda son principalmente carbonatos, hidróxidos, óxidos de $\mathrm{Ca}, \mathrm{Mg}$ y fuentes 
de Si (Alcarde 1992) y presentan diferente naturaleza química, que genera una capacidad variable de neutralización de la acidez del suelo (Chaves 1993 citado por Valerio y Molina 2012).

La fuente principal de enmienda es el carbonato de calcio que es extraído de yacimientos de rocas calizas formadas por levantamiento de pisos marinos (Flores 1999). Las rocas calizas son molidas para obtener un producto fino, que entre más pequeño mayor es su capacidad de neutralización de la acidez del suelo (Molina 1998).

Las enmiendas usualmente se aplican al suelo en forma sólida como polvo fino (Valerio y Molina 2012) sin embargo, con el uso de coadyuvantes y agentes dispersantes es posible lograr suspensiones de la enmienda en agua (Barber 1984, Peters et al. 1996). Las enmiendas en suspensión o cales líquidas son ideales para aplicación en cultivos de cero o mínima labranza donde la cal no se puede incorporar, también es de fácil manejo técnico ya que no requiere de altas cantidades lo que a su vez facilita las labores en campo y su transporte (Valerio y Molina 2012).

Las enmiendas líquidas son suspensiones alcalinizantes concentradas que pueden presentarse en forma líquida o en polvos para disolver en agua, a partir de partículas muy finas mezcladas con agua y coadyuvantes que no permiten precipitar el producto (Peters et al. 1996). Las suspensiones concentradas facilitan la manipulación cuando las partículas son muy pequeñas, principalmente cuando se realizan calibraciones de equipos de aspersión (Sánchez 2006). También se encuentran productos con formulaciones de polvos mojables, por sus siglas en inglés "wettable powder" (WP) que son productos en polvo con partículas muy finas que se mezclan con agua para formar una suspensión o dispersión (IICA 1988, Sánchez 2006).

Los aditivos o coadyuvantes son muy variables entre los productos formulados. Se toman en cuenta 2 parámetros fundamentales para generar la suspensión sólido - líquido: el tamaño de partículas y la neutralidad del coadyuvante ante el material que se utiliza como enmienda, que por lo general es carbonato de calcio (Salager 1992). Las enmiendas líquidas por lo general presentan un tamaño de partícula muy fino, que pasan entre cribas de $600-625$ mesh (Camacho et al. 2015). Estos productos tienen distintos coadyuvantes como bentonita y atapulgita (Peters et al. 1996) que principalmente se identifican en ingredientes inertes y no se especifica el componente en la etiqueta del producto. Otros productos utilizan un polisacárido extracelular llamado "goma Xanthana" que se produce por medio de fermentación de bacterias (Xanthomonas campestris), la goma suspende las partículas homogéneamente y es inerte con el $\mathrm{CaCO}_{3}$ (Valerio y Molina 2012).

Los efectos de las enmiendas en la productividad de los cultivos en suelos ácidos han demostrado excelentes resultados en diversas investigaciones, principalmente por la disminución de la acidez y una estimulación del crecimiento en las plantas (Solórzano 1974, López 1986, Gómez et al. 1996, Berettino y Loredo 2005). El encalado en suelos ácidos sembrados con maíz optimizó la producción y la vigorosidad de las plantas, así como la disponibilidad de $\mathrm{K}, \mathrm{Ca}, \mathrm{Mg}, \mathrm{Zn}$, y presentó una reducción significativa en la concentración tóxica de $\mathrm{Al}$ y $\mathrm{Mn}$ (Solórzano 1974, Gómez et al. 1996, Echeverría et al. 2001, Caires et al. 2008, Andric et al.2012). Pruebas efectuadas con enmiendas líquidas en arroz mostraron un incremento en el rendimiento y en el contenido de $\mathrm{Ca}$ en el suelo (Valerio y Molina 2012).

Existe muy poca información publicada acerca del uso de enmiendas líquidas en suelos y cultivos y la mayoría de los materiales utilizados fueron desarrollados recientemente. El cálculo de la dosis de enmienda líquida es uno de los temas que necesita ser más investigado debido a que los métodos conocidos de dosificación para productos en polvo, parece que no son aplicables para los materiales líquidos debido a aparente mayor reactividad en el suelo. El objetivo de este trabajo fue evaluar el efecto de la aplicación de 3 enmiendas líquidas en la fertilidad de un suelo, y en el crecimiento y la absorción de nutrimentos en maíz en condiciones controladas. 


\section{MATERIALES Y MÉTODOS}

El experimento se llevó acabo entre marzo y julio de 2013 en el invernadero ubicado en el Centro de Investigaciones Agronómicas (CIA) de la Universidad de Costa Rica en San Pedro de Montes de Oca.

Se utilizó un suelo clasificado como Ultisol, originario de Orosi, Cartago. El suelo se desmenuzó y se secó al aire durante 3 días. Se realizó un análisis químico completo (Cuadro 1) en el Laboratorio de Suelos y Foliares del CIA según la metodología del laboratorio de DíazRomeu y Hunter (1978). Se utilizó este suelo ya que presentó características ácidas (acidez intercambiable de 4,64 cmol(+)..$\left.^{-1}\right)$ y bajos contenidos de $\mathrm{Ca}, \mathrm{Mg}, \mathrm{K}, \mathrm{P}$ y Zn.

Cuadro 1. Análisis químico del suelo experimental.

\begin{tabular}{|c|c|c|c|c|c|c|c|c|c|c|c|c|}
\hline \multirow{2}{*}{ Suelo } & \multirow{2}{*}{$\begin{array}{c}\mathrm{pH} \\
\mathrm{H}_{2} \mathrm{O}\end{array}$} & \multicolumn{5}{|c|}{$\operatorname{cmol}(+) \cdot l^{-1}$} & \multicolumn{3}{|l|}{$\%$} & \multicolumn{3}{|c|}{$\operatorname{mg} .1^{-1}$} \\
\hline & & ACIDEZ & $\mathrm{Ca}$ & $\mathrm{Mg}$ & $\mathrm{K}$ & CICE & $\mathrm{SA}$ & $\mathrm{P}$ & $\mathrm{Zn}$ & $\mathrm{Cu}$ & $\mathrm{Fe}$ & $\mathrm{Mn}$ \\
\hline Nivel Crítico & 5,5 & 0,5 & 4 & 1 & 0,2 & 5 & $<15$ & 10 & 3 & 1 & 10 & 5 \\
\hline Orosí & 4,8 & 4,64 & 0,39 & 0,12 & 0,05 & 5,20 & 89 & 2 & 0,8 & 3 & 34 & ND \\
\hline
\end{tabular}

La investigación se realizó con fuentes de enmiendas de formulación líquida (Cal 56 y Surkal), que son suspensiones de carbonato de calcio alta fineza en agua, y de Surflow Calcio, que es un polvo mojable o dispersable que se disuelve en el agua, cuyas características químicas se presentan en el Cuadro 2. Estos 3 materiales están compuestos de carbonato de calcio que pasa por una malla de 600 mesh, que es extremadamente fina y que teóricamente los hace mucho más reactivos que los carbonatos de calcio en polvo tradicionales, que en su mayoría presentan granulometría de malla 60 mesh.

Cuadro 2. Características químicas de las enmiendas utilizadas.

\begin{tabular}{|c|c|c|c|c|c|}
\hline \multirow{2}{*}{ Enmienda } & \multicolumn{5}{|c|}{$\% *$} \\
\hline & $\mathrm{CaO}$ & $\mathrm{N}$ & EQ & EG & PRNT \\
\hline Cal 56 ${ }^{\circledR}$ & 56 & 7 & 100 & - & - \\
\hline Surkal $^{\circledR}$ & 54 & 6 & 96 & - & - \\
\hline Sur Flow Calcio ${ }^{\circledR}$ & 54 & - & 96 & 100 & 96 \\
\hline
\end{tabular}

$\mathrm{EQ}=$ Equivalente químico

$\mathrm{EG}=$ Eficiencia granulométrica

PRNT $=$ Poder relativo de neutralización total

*Datos del fabricante. 
Con las fuentes líquidas se utilizaron 2 dosis de 0,83 (a) y 1,25 (b) ml.maceta ${ }^{-1}$, así mismo se utilizó una fuente de polvo mojable a 2 dosis de 4,16 (a) y 8,32 (b) g.maceta ${ }^{-1}$ (Cuadro 3).

Cuadro 3. Tratamientos de enmiendas utilizadas.

\begin{tabular}{llll}
\hline Tratamiento & Código & $\begin{array}{l}\text { Dosis } \\
\text { maceta } 11 .\end{array}$ & $\begin{array}{l}\text { Dosis } \\
\text { maceta 0,5 1. }\end{array}$ \\
\hline Testigo & Testigo & $0 \mathrm{ml}$ & $0 \mathrm{ml}$ \\
& C56 a & $0,83 \mathrm{ml}$ & $0,42 \mathrm{ml}$ \\
Cal 56 & C56 b & $1,25 \mathrm{ml}$ & $0,63 \mathrm{ml}$ \\
& SC a & $4,16 \mathrm{~g}$ & $2,1 \mathrm{~g}$ \\
Surflow Calcio & SC b & $8,32 \mathrm{~g}$ & $4,2 \mathrm{~g}$ \\
& SK a & $0,83 \mathrm{ml}$ & $0,42 \mathrm{ml}$ \\
Surkal & SK b & $1,25 \mathrm{ml}$ & $0,63 \mathrm{ml}$ \\
\hline
\end{tabular}

Las enmiendas se diluyeron en un volumen de $400 \mathrm{ml}$ de agua para cada dosis de acuerdo con el tratamiento, y se aplicaron en forma de lámina de riego de manera homogénea y que impregnara todo el volumen de la maceta que contenía 1000 $\mathrm{ml}$ de suelo. Se dejó reaccionar la enmienda durante 7 días y posteriormente se sembraron 5 semillas de maíz (Zea mays L.) de la variedad Diamantes como planta indicadora. A los 7 días después de siembra (dds) se raleó a 4 plantas en cada maceta. Las dosis por maceta se calcularon con base en la densidad de siembra de maíz de 48000 plantas.ha $^{-1}$, de acuerdo con Elizondo y Boschini (2001). Con base en esta densidad de siembra de maíz y con una dosis por planta, las cantidades utilizadas en este experimento serían equivalentes a 10 y 15 1.ha- ${ }^{-1}$ para Cal 56 y Surkal, y a 50 y $100 \mathrm{~kg} \mathrm{ha}^{-1}$ para el Surflow Calcio, cabe indicar que no existe un método para determinar la dosis de enmiendas líquidas y en polvo mojable a base de $\mathrm{CaCO}_{3}$, estas dosis fueron propuestas con base en recomendaciones de la etiqueta de los productos, pruebas exploratorias realizadas por los autores de este trabajo, y publicaciones recientes de enmiendas líquidas (Valerio y Molina 2012, Camacho et al. 2015).

Para las enmiendas sólidas en polvo existen diferentes métodos de cálculo de dosis, como el de reducir la saturación de acidez, o el de multiplicar el valor de acidez intercambiable por un factor (Espinosa y Molina 1999). Sin embargo, para enmiendas líquidas ninguno de estos métodos se puede utilizar debido a que por su elevada fineza en comparación con las enmiendas tradicionales, su reactividad en el suelo parece ser mucho más alta que los materiales en polvo de menor granulometría. La información que existe acerca del uso de enmiendas líquidas es muy escasa, y aunque los fabricantes recomiendan utilizarlas en dosis desde 20 a 100 1.ha ${ }^{-1}$, todavía no hay suficiente evidencia experimental para estimar la dosis.

Una vez sembradas las plantas, las macetas se introdujeron en tinas plásticas con una lámina de agua para efectuar el riego por capilaridad a través de un filtro de algodón ubicado en un agujero de la parte de abajo de la maceta y que se sumergió en la tina. Se aplicó una concentración de $50 \mathrm{mg} . \mathrm{l}^{-1}$ de $\mathrm{N}$ al agua de riego en la tina. Posteriormente a los 15 días después de siembra (dds) se aplicó a cada maceta 2 g de Fosfato Diamónico (18-46-0) y a los 29 dds $1 \mathrm{~g}$ de Cloruro de Potasio (0-0-60) ambos en polvo previamente molido.

Adyacente a las macetas con plantas se colocó otro ensayo en macetas de $500 \mathrm{ml}$ del mismo suelo para evaluar el efecto de las enmiendas en la acidez y pH a través del tiempo. En éstas se aplicó proporcionalmente las mismas dosis propuestas anteriormente (Cuadro 3), pero sin sembrar maíz, con el objetivo de tomar muestras para la medición de $\mathrm{pH}$ y acidez intercambiable en el tiempo. Se utilizó una lámina de riego de $225 \mathrm{ml}_{\text {. }}$ aceta $^{-1}$ para aplicar a todo el volumen de suelo con las enmiendas disueltas. En las macetas de $500 \mathrm{ml}$ se tomaron muestras de $30 \mathrm{~g}$ de suelo a $\operatorname{los} 8,14,19,27,35,45$ y 60 días después de la 
aplicación (dda). En estas muestras se realizaron análisis de $\mathrm{pH}$ en agua y acidez intercambiable, de acuerdo con la metodología propuesta por Henríquez y Cabalceta (2012).

Se hicieron mediciones del grosor de tallo y longitud de parte aérea, a $\operatorname{los} 8,22,38$ y 45 después de siembra. El grosor de tallo se midió con un vernier a una altura de $1 \mathrm{~cm}$ de la superficie del suelo, y la longitud con una cinta métrica desde la superficie del suelo hasta la punta de la hoja más larga de la planta.

Las plantas se cosecharon a los 45 dds extrayendo la parte aérea y las raíces. Se midió con una cinta métrica la longitud de raíz desde la parte inferior del tallo hasta el meristemo apical de la raíz más larga. Las raíces y la parte aérea se lavaron con agua destilada y se empacaron en bolsas de papel y se llevó a peso constante en un horno a $75^{\circ} \mathrm{C}$ durante 48 horas, y se midió el peso seco de raíz y parte aérea. Para cuantificar el efecto del encalado en las raíces se determinó la densidad de raíces y densidad longitudinal. La densidad de raíces se calculó estimando el peso seco de las raíces y se dividió entre el volumen de la maceta (1000 ml). Así mismo, para estimar la densidad longitudinal se dividió la longitud de las raíces entre el volumen de la maceta según la metodología de Pire (1985).

Se hizo un análisis químico de N, P, Ca, $\mathrm{Mg}, \mathrm{K}, \mathrm{S}, \mathrm{Fe}, \mathrm{Cu}, \mathrm{Zn}, \mathrm{Mn}$ y $\mathrm{B}$ en las muestras secas y molidas de la parte aérea de las plantas. Además, se recolectaron muestras de suelo de cada maceta después de la cosecha de plantas (45 dds) para análisis químico de $\mathrm{Ca}, \mathrm{Mg}, \mathrm{K}, \mathrm{P}, \mathrm{Zn}$, $\mathrm{Cu}, \mathrm{Fe}, \mathrm{Mn}$, Acidez Intercambiable y $\mathrm{pH}$ en el Laboratorio de Suelos y Foliares en el CIA (DíazRomeu y Hunter 1978).

\section{Análisis de datos}

El diseño experimental utilizado fue un irrestricto al azar con 7 tratamientos y 4 repeticiones, donde cada maceta representó una unidad experimental. Para el análisis de los datos, los parámetros medidos en las 4 plántulas se promediaron para obtener un dato representativo. Los datos promedios tomados a los 8, 22, 38, 45 dds de las variables (grosor de tallo, longitud de planta) se reunieron con el índice llamado "índice de área bajo la curva (abc)", con el fin de reunir información de las evaluaciones tomadas en el tiempo. Esta prueba consistió en calcular el área de un trapecio entre 2 fechas de evaluación (Ecuación 1). La ecuación se calculó entre el día $8-22,22-38$ y $38-45$ para el grosor de tallo y longitud de planta en cada fecha y una vez obtenido el área de los trapecios, se sumaron las 3 áreas para determinar el abc.

$$
A=\frac{(B+b) \times a}{2}
$$

Ecuación 1

Donde:

$\mathrm{A}=$ área de un trapecio.

$\mathrm{B}=$ lectura de variable en la última fecha de evaluación.

$\mathrm{b}=$ lectura de variables en la primera fecha de evaluación.

$a=$ tiempo transcurrido entre la primera medición y segunda medición (días).

La acidez y el $\mathrm{pH}$ se analizaron estadísticamente a $\operatorname{los} 8,14,19,27,35,45$ y 60 dda, con el fin de comparar la concentración en las fechas evaluadas y determinar diferencias entre los tratamientos de cales. Así mismo, se procedió a realizar una curva de acidez y de $\mathrm{pH}$ para observar el comportamiento en el tiempo en cada tratamiento. Los nutrimentos foliares y de suelos fueron procesados mediante análisis estadísticos para cada uno de los nutrimentos, con el fin de encontrar diferencias entre los tratamientos evaluados.

Para el análisis de curvas de respuesta a encalado, análisis químico de suelo, concentración de nutrimentos foliar, abc, densidad de raíces y longitudinal; fueron procesados mediante un análisis de varianza con el programa 
estadístico ANAWIN. Posteriormente se sometieron al cálculo de separaciones de medias, mediante la prueba de diferencias mínimas significativas (d.m.s) $(\alpha=0,01)$ para determinar diferencias entre tratamientos.

\section{RESULTADOS Y DISCUSIÓN}

\section{Efecto de las enmiendas en la acidez del suelo en el tiempo}

El efecto de las enmiendas en la acidez intercambiable y el $\mathrm{pH}$ del suelo fueron evaluados mediante la medición periódica de estas variables en un período de 60 días después de aplicadas.

En general, las enmiendas tuvieron un efecto significativo $(\alpha=0,01)$ en la disminución de la acidez intercambiable en comparación al testigo (Figura 1). La curva de reacción de la acidez intercambiable producida por las enmiendas disminuyó en forma proporcional a la dosis aplicada. Efectos similares encontraron Molina y Rojas (2005) con aplicaciones de diferentes fuentes de carbonato de calcio en naranja Valencia.
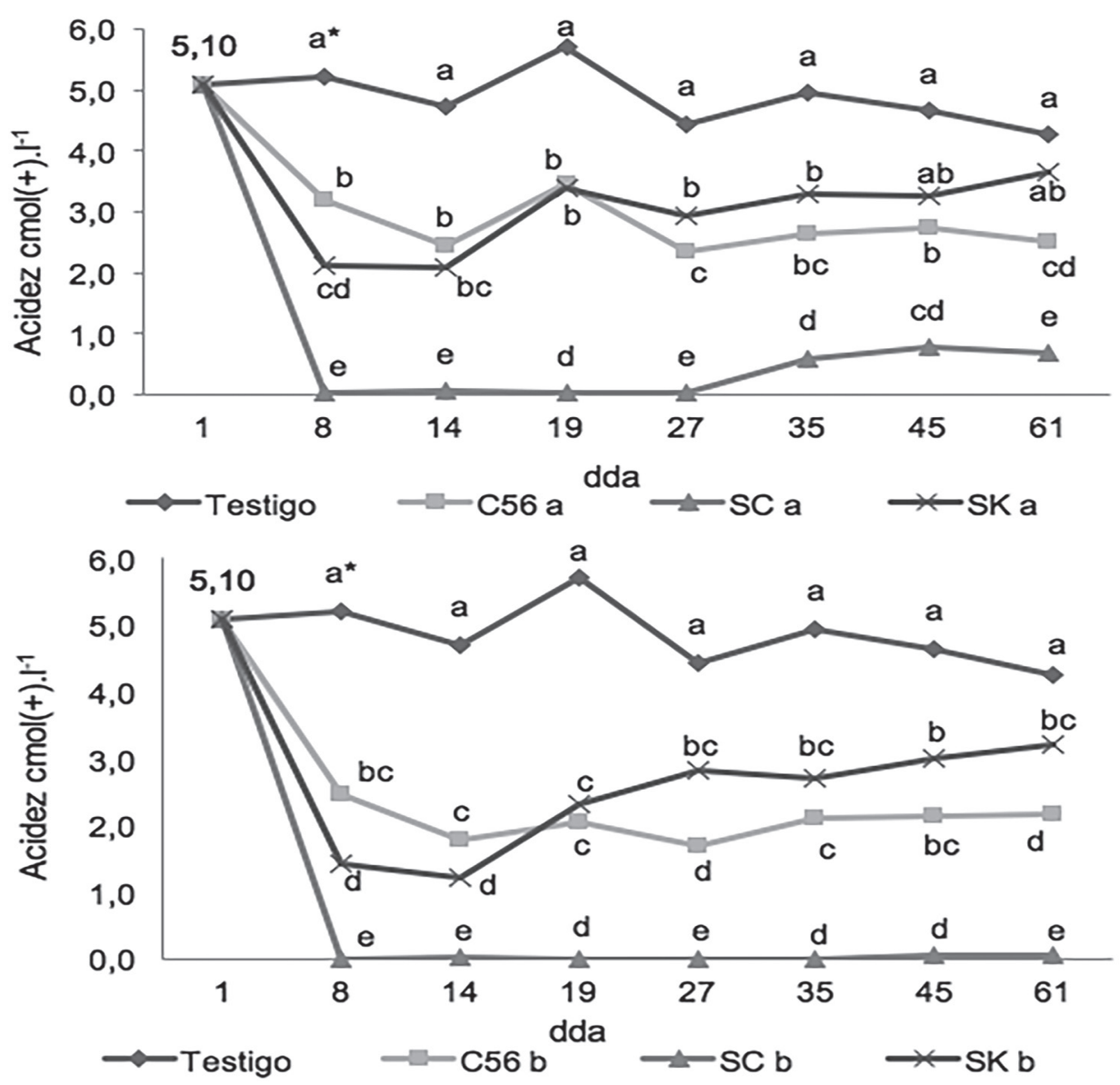

Fig. 1. Curva de respuesta de acidez intercambiable en el tiempo a la aplicación de enmiendas líquidas en un Ultisol.

* Medias seguidas por la misma letra no se consideran distintas según prueba de d.m.s $(\alpha=0,01)$. 
A los 8 dda, las 2 dosis de SK disminuyeron la acidez intercambiable en 60 y $73 \%$ con respecto al testigo sin cal (Figura 1). El tratamiento C56 también disminuyó la acidez en 39 y $53 \%$, respectivamente, con respecto al testigo. La reducción máxima de la acidez para C56 se presentó a los 14 dda, donde obtuvo una disminución de 48 y $62 \%$ con ambas dosis, respectivamente. Las enmiendas líquidas SK y C56 presentaron un efecto marcado de reducción de acidez hasta los 27 dda (Figura 1).

El efecto de las 2 enmiendas líquidas sobre la acidez del suelo fue muy rápido, a los 8 dda ya presentaba una reacción inmediata y fue más evidente a los 14 dda. A partir de ahí la acidez intercambiable comenzó a subir, probablemente por la fuerte capacidad buffer que caracteriza a los Ultisoles debido a su mineralogía de arcillas de carga variable dependiente de pH (Espinosa y Molina 1999). El efecto residual de la enmienda también depende de la dosis aplicada, y en el caso de este estudio la dosis de enmienda líquida si bien tuvo un efecto significativo sobre la acidez intercambiable, no fue suficiente para mantener la reducción de la acidez al nivel inicial durante el período del ensayo (61 días). La rápida reacción de las enmiendas líquidas probablemente se debe a su alta fineza (malla 600 mesh), que causaría una reacción química casi inmediata sobre la acidez del suelo (Camacho et al. 2015), contrario a las enmiendas en polvo tradicionales que por lo general reaccionan en un período de 3 a 6 meses después de aplicadas, de acuerdo con su PRNT (Espinosa y Molina 1998, Molina y Rojas 2005), esto debido a que su tamaño de partículas (malla 60 mesh) es menor que el de las enmiendas líquidas.

El tratamiento SK a partir del día 19 dda presentó un aumento exponencial en la acidez de $0,36 \mathrm{cmol} . \mathrm{l}^{-1}$ hasta el día 60 después de aplicado en ambas dosis, mientras que el tratamiento C56 presentó un aumento exponencial de $0,04 \mathrm{cmol}^{\mathrm{l}^{-1}}$ en ambas dosis. Esto predice que la C56 mantuvo una mejor estabilidad de reacción en el tiempo superior al producto SK. A los 60 dda la C56 con la dosis más alta mostró diferencia significativa $(\alpha=0,01)$ en la disminución de la acidez en comparación a la dosis más baja del mismo producto. $\mathrm{Si}$ bien la concentración de $\mathrm{Ca}$ y $\mathrm{N}$ es casi igual en ambas enmiendas líquidas (Cuadro 2), C56 fue más eficiente en reducir la acidez del suelo a corto plazo quizás debido a la presencia de la goma Xanthana como agente coadyuvante, que permite una mayor adherencia y persistencia de la enmienda en el suelo. Valerio y Molina (2012) encontraron que C56 mostró una reacción rápida y una alta persistencia al aplicarlo en un Ultisol de Los Chiles, que mejoró levemente el contenido de Ca en el suelo.

El tratamiento SC presentó la mayor reducción de la acidez intercambiable del suelo en comparación con las otras 2 enmiendas líquidas. Este producto es un polvo mojable que se disuelve en agua y se aplica en forma líquida. El SC disminuyó la acidez desde 5,10 a 0,01 cmol. $\mathrm{l}^{-1}$ con la dosis más alta (SC b) durante los 60 días evaluados, mientras que con la dosis más baja (SC a) obtuvo el mismo comportamiento pero en un tiempo más corto (35 dda) donde mostró un incremento en la acidez de 0,01 a $0,58 \mathrm{cmol}^{-1} \mathrm{I}^{-1}$. El mayor efecto de la enmienda en polvo mojable sobre la acidez del suelo fue debido a que se utilizaron dosis más altas, esto es, 4,16 y 8,32 g.maceta ${ }^{-1}$. Cuadro 3, en comparación con las enmiendas líquidas $(0,83$ y 1,25 ml.maceta $\left.{ }^{-1}\right)$.

Se encontraron diferencias altamente significativas $(\alpha=0,01)$ en el efecto de las enmiendas líquidas con el aumento de $\mathrm{pH}$ del suelo (Figura 2). El producto $\mathrm{SC}$ en la dosis más alta aumentó el pH de 4,5 a 7,8 hasta el día 14 después de aplicado. El pH presentó una correlación inversamente proporcional con la reducción de la acidez, lo cual es un comportamiento normal al efecto del encalado (Bertsch 1995, Molina 1998, Braeuner et al. 2005, Henríquez y Cabalceta 2012). 

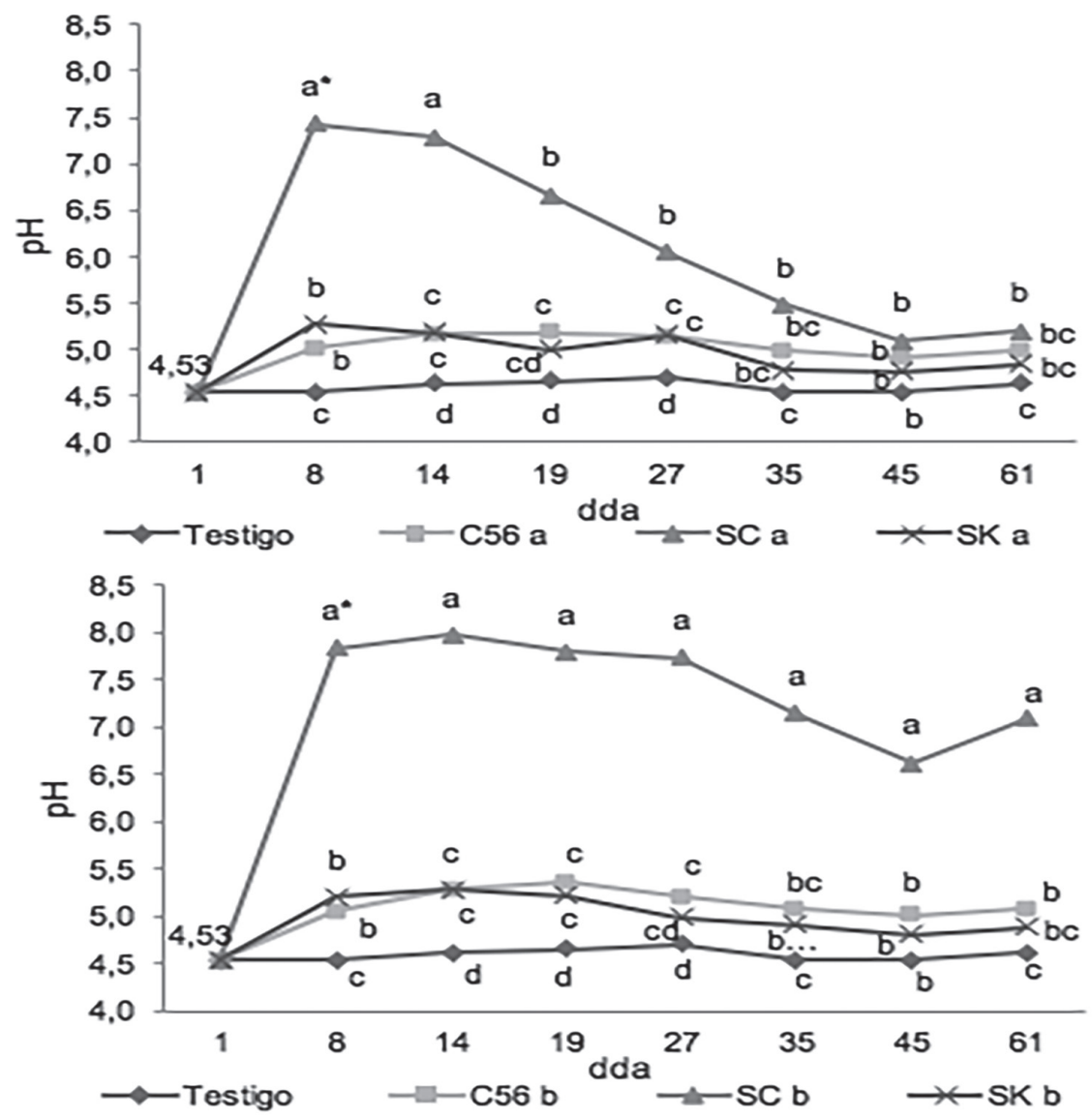

Fig. 2. Curva de pH en el tiempo a la aplicación de enmiendas líquidas en un Ultisol.

*Medias seguidas por la misma letra no se consideran distintas según prueba de d.m.s $(\alpha=0,01)$.

Hasta los 35 dda, los tratamientos de SK y C56 en ambas dosis, y el SC en la dosis más baja, presentaron un $\mathrm{pH}$ superior al testigo con diferencias significativas (Figura 2). A partir de los $35 \mathrm{dda}$, el $\mathrm{pH}$ del testigo fue similar a estos tratamientos, lo que muestra el fuerte poder buffer de este suelo que tiende a volver a su pH original. Datos similares encontraron Camacho et al. (2015) y Alvarado et al. (2010) donde determinaron que la capacidad buffer presente en Ultisoles, pueden resistir cambios fuertes de $\mathrm{pH}$ aún después de la aplicación de enmiendas.

El tratamiento de enmienda en polvo SC con la dosis más alta presentó el mayor incremento de $\mathrm{pH}$ (Figura 2), con diferencias significativas respecto a los demás tratamientos, y mantuvo un pH alto después de 60 días de aplicado. 
Este tratamiento también mostró la mayor reducción de la acidez intercambiable.

En todos los tratamientos evaluados el efecto sobre la acidez del suelo fue muy rápido, aunque su efecto residual tiende a reducirse a partir de los 45 dda. La excepción fue la dosis más alta de SC, que mantuvo baja la acidez y más alto el $\mathrm{pH}$ durante el período de 60 días de evaluación. Resultados similares con enmiendas líquidas han sido reportados recientemente por Camacho et al. (2015).
Efecto de las enmiendas en el contenido de nutrimentos en el suelo

La aplicación de enmiendas causó cambios positivos en la fertilidad del suelo al aumentar el pH, disminuir la acidez intercambiable y la saturación de acidez, e incrementar el contenido de $\mathrm{Ca}$ al final del experimento, con diferencias significativas entre tratamientos (Cuadro 4). 


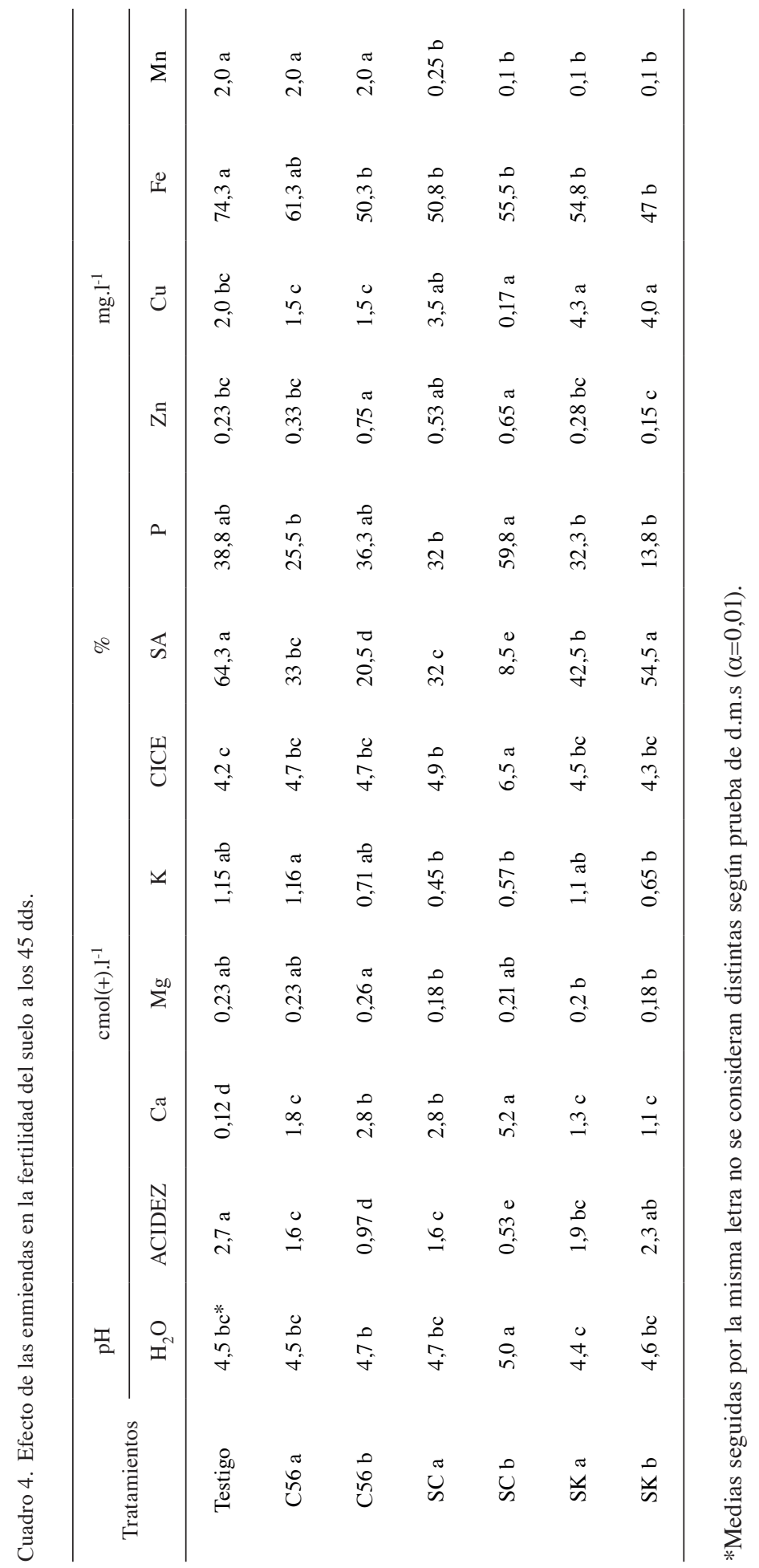


La aplicación de enmiendas redujo la acidez intercambiable y la saturación de acidez. La dosis más alta de la enmienda en polvo mojable (SC) presentó los valores más bajos de acidez intercambiable y saturación de acidez, con diferencias significativas respecto al resto de tratamientos. Los tratamientos SC y C56 con la dosis más alta presentaron los valores más bajos de saturación de acidez, con 8,5 y 20,5\%, respectivamente. Los resultados coinciden con Camacho et al. (2015), que encontraron una reducción significativa de la acidez del suelo con aplicaciones de enmiendas líquidas en un Ultisol cultivado de sorgo en invernadero. Salas et al. (1996), y Molina y Rojas (2005), encontraron que la aplicación de enmiendas a base de carbonatos de calcio disminuyeron la acidez intercambiable y la saturación de acidez en Ultisoles de la zona norte del país.

El tratamiento de la dosis más alta de SC redujo la acidez desde 2,7 a $0,53 \mathrm{cmol}(+) . l^{-1}$ en comparación al testigo. No obstante la curva de acidez intercambiable a través del tiempo, mostró concentraciones inferiores al suelo analizado a los 45 dda con plantas de maíz (Figura 1). Probablemente este efecto sea porque el suelo utilizado en la curva de reactividad fue una prueba directa con las enmiendas líquidas, mientras que los suelos analizados a los $45 \mathrm{dds}$ presentaban la misma concentración de enmiendas pero en macetas cultivadas con maíz y fertilizadas con N. Posiblemente la liberación de $\mathrm{H}^{+}$producto del intercambio entre formas catiónicas en la interacción raíz-suelo en la rizosfera (forma compensatoria), y provenientes de la nitrificación del $\mathrm{NH}_{4}{ }^{+}$del fertilizante, produciría el efecto acido del suelo (Bertsch 1995, Zapata 2004).

$\mathrm{El}$ efecto de las enmiendas en el $\mathrm{pH}$ fue mínimo, sólo la dosis más alta de SC logró subir el $\mathrm{pH}$ de 4,5 a 5,0 a los 45 dds, con diferencias significativas respecto a los demás tratamientos. Este efecto podría explicarse por el alto poder buffer que presentan estos suelos, pues hace que el $\mathrm{pH}$ no aumente en forma significativa aún con la aplicación de enmiendas, debido a la mineralogía de arcillas de carga variable dependiente de pH que caracteriza a estos suelos ácidos (Espinosa y Molina 1999). También es probable que el efecto acidificante de la aplicación de nitrato de amonio durante la ejecución del experimento pudo haber causado la liberación de iones $\mathrm{H}^{+}$ que no permitieron que las enmiendas líquidas aumentaran de forma significativa el $\mathrm{pH}$, ya que el suelo fue fertilizado durante todo el periodo con $\mathrm{N}$ en la lámina de agua.

El $\mathrm{P}$ y $\mathrm{K}$ presentaron concentraciones altas en todos los tratamientos a los $45 \mathrm{dds}$, contrario al inicio del experimento donde sus niveles eran muy bajos. Esto se debe a que se aplicaron DAP y $\mathrm{KCl}$ a los 15 y 29 dds aspecto que aumentó las concentraciones de estos nutrimentos en el suelo. Se aplicó el fertilizante debido a que las plantas presentaron síntomas de deficiencia. La aplicación de enmiendas disminuyó los contenidos de K y P. En el caso del $\mathrm{K}$ probablemente debido al ya conocido efecto de antagonismo causado por la aplicación de $\mathrm{Ca}$ (Bertsch 1995). La fijación de una parte del $\mathrm{P}$ disponible como fosfatos de calcio podría ser la causa de la disminución en su contenido al aplicar las enmiendas, efecto que se podría acelerar debido a la fuerte reactividad de las enmiendas líquidas. Todas las enmiendas aumentaron de forma significativa el contenido de $\mathrm{Ca}$, aunque el efecto más pronunciado fue con la enmienda en polvo mojable y la dosis más alta de C56. La dosis alta de SC presentó el mayor contenido de $\mathrm{Ca}$ a los 45 dds, con un aumento de 5 cmol(+). $1^{-1}$ en su concentración con respecto al testigo. La concentración de calcio correlacionó inversamente con la curva de acidez $\left(\mathrm{r}^{2}=-0,94\right)$ y proporcionalmente con el $\mathrm{pH}\left(\mathrm{r}^{2}=0,94\right)$ de acuerdo con la concentración de acidez y $\mathrm{pH}$ a los 45 dda evaluados en la curva de reactividad de enmiendas. La aplicación de enmienda por lo general incrementa los contenidos de $\mathrm{Ca}$ en 
el suelo tal y como ha sido documentado por muchos autores (Salas et al. 1996, Espinosa y Molina 1999, Furtini et al. 1999, Molina y Rojas 2005, Camacho et al. 2015). No hubo efecto de las enmiendas en el $\mathrm{Mg}$ probablemente porque ninguna de ellas contiene este elemento.

\section{Efecto de las enmiendas líquidas en el contenido foliar de nutrimentos}

Se encontraron diferencias mínimas significativas $(\alpha=0,01)$ para la mayoría de los nutrimentos en la planta a excepción del $\mathrm{Fe}$ (Cuadro 5). El N no mostró diferencias estadísticas entre los tratamientos de enmiendas líquidas, pero si superaron al testigo, ya que la planta disminuyó la absorción de este elemento en ausencia de enmienda. Estos resultados concuerdan con Muena et al. (2010) que encontraron que en los suelos encalados aumentaron la absorción de $\mathrm{N}$ en plantas de Oenothera affinis cuando recibieron fertilización nitrogenada. 


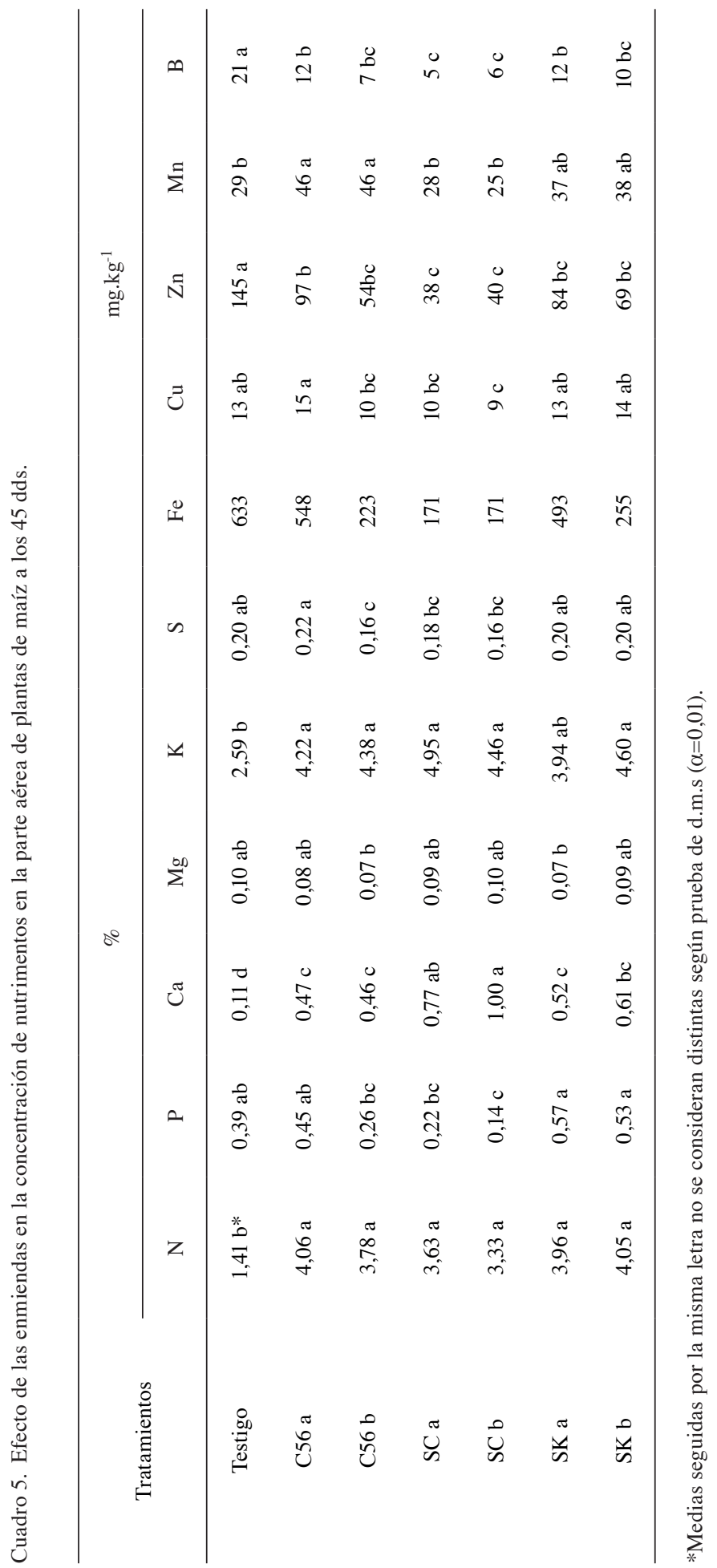


La concentración de $\mathrm{P}$ foliar disminuyó con la dosis de enmienda, al igual que ocurrió en el suelo. La enmienda de polvo mojable (SC) presentó la concentración más baja de $\mathrm{P}$ foliar, con diferencias significativas con la dosis más alta, que probablemente causó una disminución en la disponibilidad de $\mathrm{P}$ en el suelo y redujo su absorción por la planta. Dosis altas de enmienda pueden causar una disminución en la disponibilidad de $\mathrm{P}$ debido a la formación de fosfatos de calcio insolubles (Bertsch 1995, Espinosa y Molina 1999, Zapata 2004). El efecto podría ser inmediato en el caso de enmiendas líquidas que reaccionan en corto plazo como ya se demostró con los datos de las Figuras 1 y 2.

Hubo un incremento en la concentración de $\mathrm{K}$ con la aplicación de enmiendas. La reducción de la toxicidad de Al y el incremento de materia seca que causaron las enmiendas, favorecieron la absorción de nutrimentos como el K y N. Los resultados coinciden con Manetti y Fernandes (2008) que encontraron que aplicaciones de enmiendas en cultivo de maíz mejoraron la absorción de K. La aplicación de enmiendas también aumentó la concentración de $\mathrm{Ca}$ foliar, resultado que era de esperar debido al suministro de Ca por parte de las enmiendas. La aplicación de enmiendas líquidas aumentó la absorción de Ca y K en plantas de sorgo cultivadas en un Ultisol en invernadero (Camacho et al. 2015).

Las enmiendas no tuvieron ningún efecto en el contenido de $\mathrm{Mg}$ foliar el cual presentó niveles de deficiencia en todos los tratamientos, síntomas que fueron evidentes en las plantas con la presencia de una clorosis intervenal (Figura 3). El contenido inicial de $\mathrm{Mg}$ en el suelo fue de 0,12 $\operatorname{cmol}(+) . l^{-1}$, nivel que se considera muy bajo y que probablemente explica los síntomas de deficiencia encontrados en las hojas del maíz.

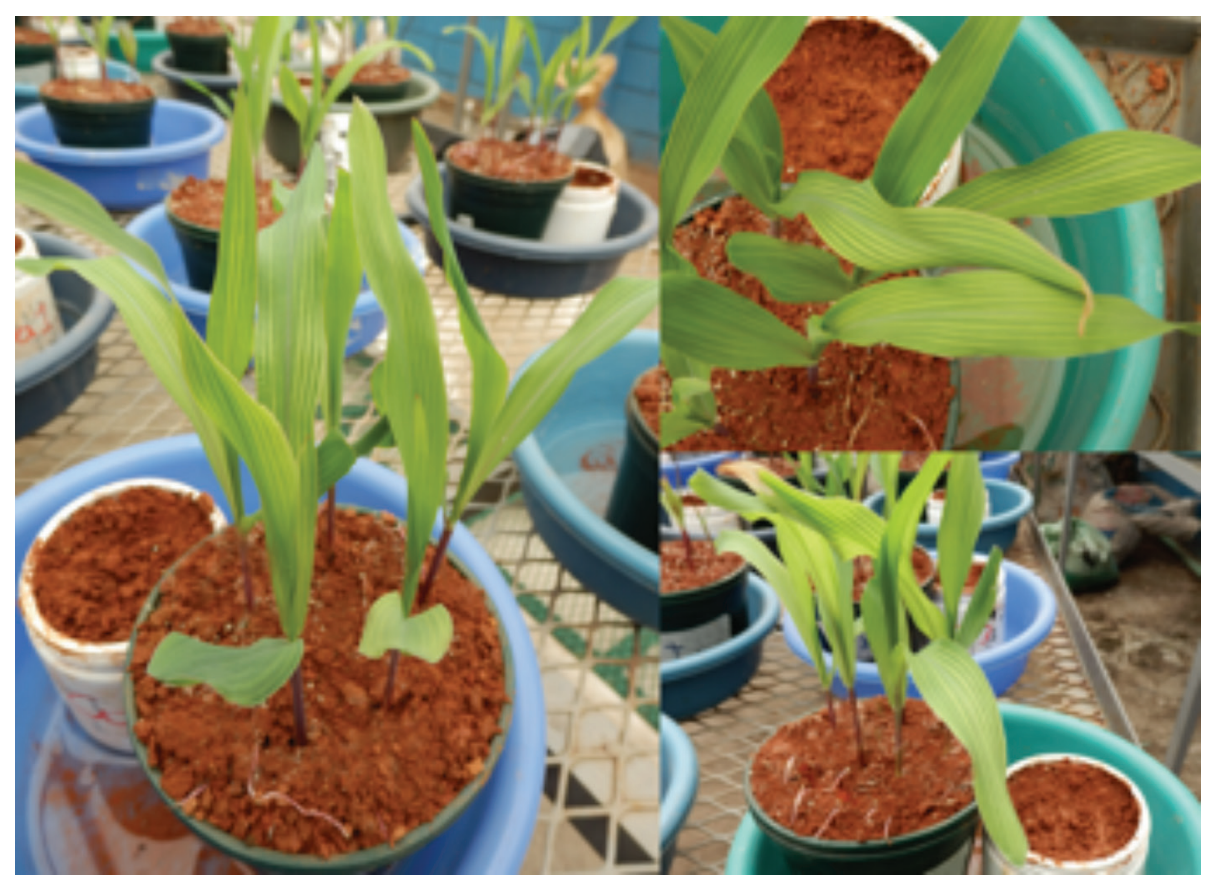

Fig. 3. Clorosis intervenal por deficiencia de Mg en plantas de maíz a los 19 dds. 
Entre los micronutrimentos hubo una disminución en los contenidos de Zn y B por la aplicación de enmiendas, con diferencias significativas con respecto al testigo, aspecto que mostró más severidad en los tratamientos de enmienda en polvo mojable que aportaron las dosis más altas y que tuvieron mayor efecto en reducir la acidez del suelo y subir el pH. El Fe también disminuyó aunque no hubo diferencias significativas. El incremento del $\mathrm{pH}$ y del contenido de $\mathrm{Ca}$ en el suelo puede disminuir la disponibilidad de micronutrimentos (Bertsch 1995, Espinosa y Molina 1999).

\section{Efecto de las enmiendas líquidas en el crecimiento de plantas de maíz}

Todos los tratamientos de enmiendas superaron al testigo con la variable de índice de grosor del tallo (Figura 4). Los mejores tratamientos fueron C56 con la dosis más alta y SC con ambas dosis. Los resultados mostraron concordancia con la curva de reactividad de las enmiendas, lo que explicaría el crecimiento en el diámetro de la plantas. La neutralización rápida de la acidez del suelo y el suministro de Ca favoreció el crecimiento de las plantas, incrementado el grosor del tallo. Alvarado y Fallas (2004) en plantas jóvenes

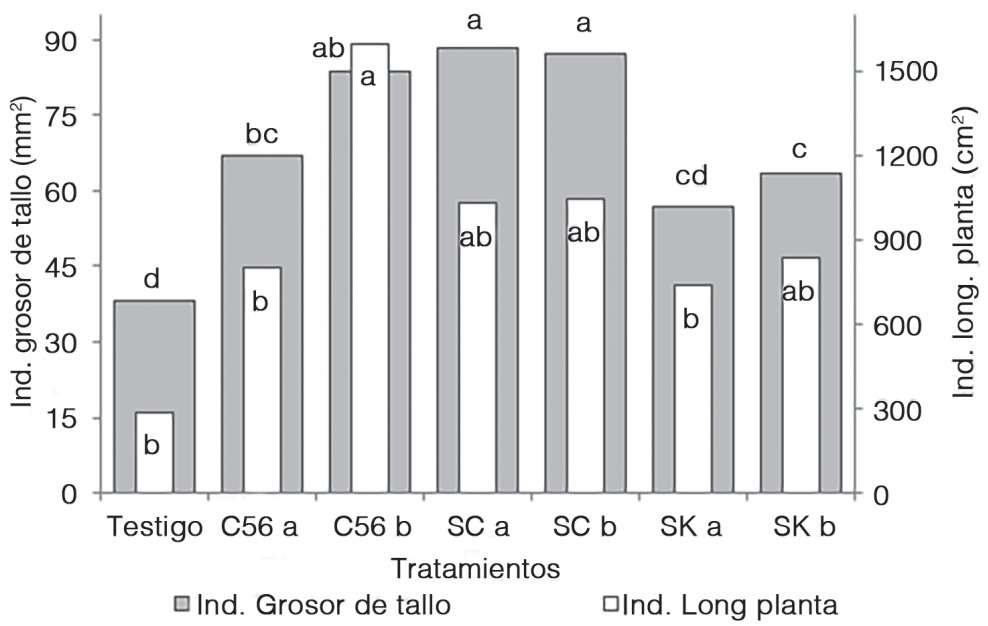

Fig. 4. Efecto de la aplicación de enmiendas en el Índice de área bajo la curva en grosor de tallo y longitud de la planta de maíz. *Medias seguidas por la misma letra no se consideran distintas según prueba de d.m.s $(\alpha=0,01)$.

de teca encontraron que la aplicación de $\mathrm{CaCO}_{3}$ aumentó el diámetro de los árboles.

Con la variable de índice de área bajo la curva en la longitud de plantas, sólo el tratamiento de C56 en la dosis más alta presentó diferencias significativas con respecto al testigo, mientras que el resto de tratamientos no mostró diferencias. Este producto tiene $7 \%$ de $\mathrm{N}$ en forma de urea lo cual podría haber favorecido el crecimiento del maíz.
La mayoría de los tratamientos de enmienda presentaron valores muy superiores al testigo en peso seco de tallo y raíces. Sin embargo, el análisis estadístico mostró pocas diferencias significativas, probablemente debido a la gran variabilidad entre repeticiones que aumentó el error estadístico (Figura 5). Sólo el tratamiento SK en dosis alta presentó diferencias significativas con el peso seco del tallo, y el SC en dosis alta con el peso seco de raíces. Los resultados con estas 


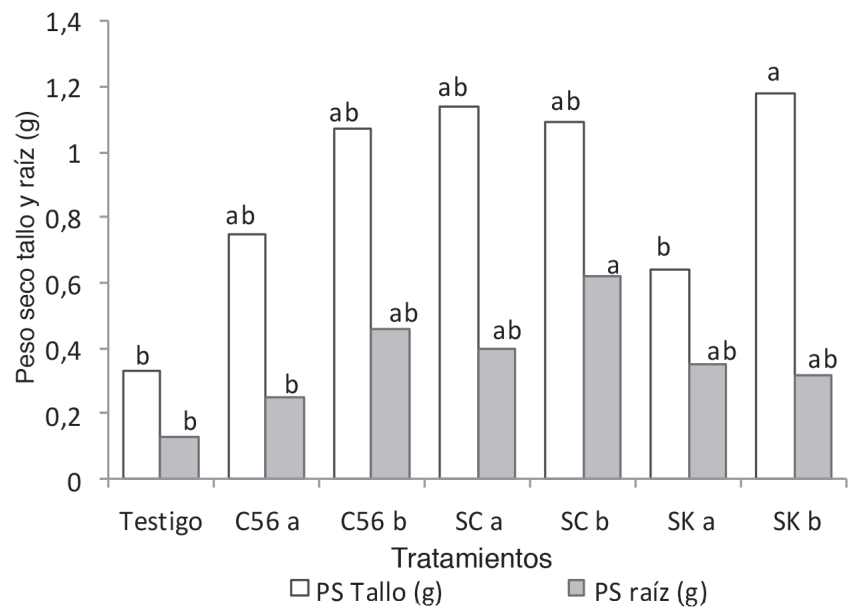

Fig. 5. Efecto de la aplicación de enmiendas en el peso seco de tallo y raíz en maíz.

*Medias seguidas por la misma letra no se consideran distintas según prueba de d.m.s $(\alpha=0,01)$.

variables concuerdan con Caires et al. (2002) quienes encontraron una mayor biomasa de maíz en conjunto con un aumento en la redistribución de las raíces al adicionar fuentes de carbonato de calcio en Oxisoles. Soratto y Crusciol (2008) encontraron un incremento en la producción de biomasa en arroz como consecuencia de la aplicación de cal dolomita. Valerio y Molina (2012) encontraron respuesta significativa en el rendimiento de arroz con la aplicación C56 en dosis en un Ultisol de Los Chiles.

La densidad longitudinal fue mayor en los 2 tratamientos de C56, con diferencias significativas con respecto al testigo y a la mayoría de resto de tratamientos (Figura 6). Todos los tratamientos de enmiendas superaron al testigo en densidad de raíces, y la dosis más alta de SC presentó la mayor densidad.

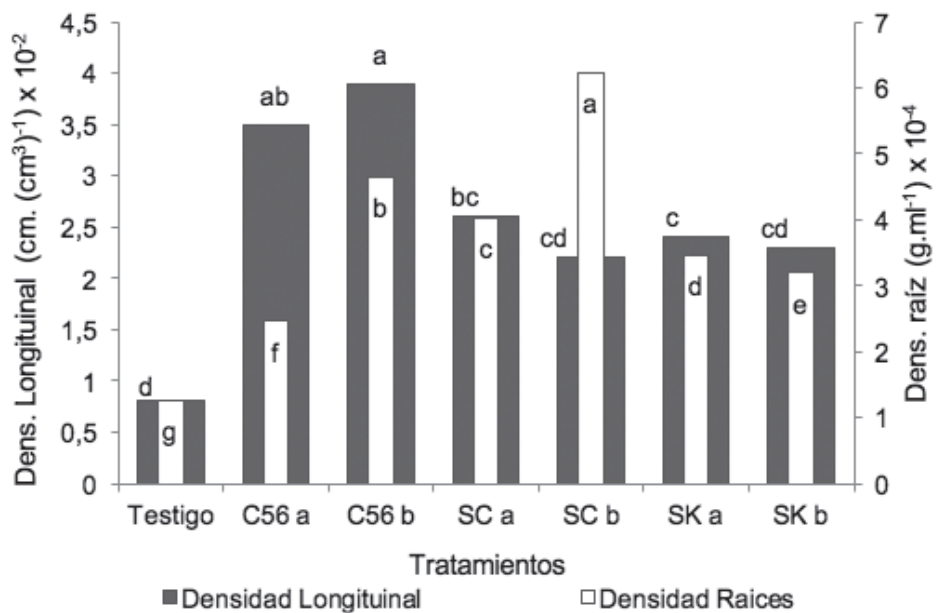

Fig. 6. Efecto de la aplicación de enmiendas en la densidad longitudinal y densidad de raíz en maíz. *Medias seguidas por la misma letra no se consideran distintas según prueba de d.m.s $(\alpha=0,01)$. 
Los tratamientos SC 50 y 100 kg.ha ${ }^{-1}$ obtuvieron el mayor desarrollo de las plantas de maíz y la mayor disminución de la acidez en la curva de reactividad de las enmiendas. Sin embargo, las observaciones en invernadero mostraron que el crecimiento de las raíces se efectuó en los primeros $8 \mathrm{~cm}$, lo que respalda la mala distribución de la cal en estos tratamientos. Posiblemente este efecto sea causa del riego efectuado en los tratamientos ya que este fue por capilaridad, esto determinaría que el riego no permitió que la cal infiltrara efectivamente en el volumen de suelo. No obstante, la C56 pudo superar el problema de infiltración de la cal, posiblemente por el efecto de la goma Xanthana, lo cual pudo ayudar en distribuir uniformemente el producto en el volumen de suelo.

La densidad longitudinal explicaría el efecto de las cales en el crecimiento de las plantas y su absorción de nutrimentos. Esta forma de obtención de nutrimentos está bien documentado por diversos autores (Clarkson y Hanson 1980, Lynch y Van Beem 1993, Villalobos 2001) al evidenciar la relación área.volumen ${ }^{-1}$ de la raíz. Los resultados coinciden con Tissi et al. (2004) y Azevedo et al. (2011) donde encontraron respuesta en la captación de nutrimentos con la aplicación de encalado en maíz, lo cual mejoró la captación de $\mathrm{P}, \mathrm{Ca}, \mathrm{Mg}, \mathrm{S}$ y no alteraron la concentración de $\mathrm{Cu}, \mathrm{Fe}, \mathrm{Mn}$ y $\mathrm{Zn}$ en el suelo ni el tejido vegetal.

\section{CONCLUSIONES}

Las enmiendas líquidas presentaron una reacción muy rápida en la neutralización de la acidez intercambiable y en el $\mathrm{pH}$, pero su efecto residual fue de corto plazo con excepción de la enmienda en polvo mojable que controló muy bien la acidez durante todo el período el ensayo. También mejoraron la fertilidad del suelo, principalmente en la reducción de la acidez intercambiable y la saturación de acidez y en el incremento del contenido de $\mathrm{Ca}$.

Las enmiendas líquidas favorecieron el crecimiento del maíz, el peso seco de la biomasa total, la longitud y densidad de raíces, condiciones que muestran que podrían ser una opción de manejo de la acidez del suelo en situaciones donde se necesita una reacción inmediata de la enmienda, y en cultivos de ciclo corto. La curva de reactividad indica detalladamente el comportamiento de reacción de las dosis de cales líquidas en el tiempo y su eficiencia en la disminución de la acidez. Las dosis evaluadas de cales disminuyen la acidez eficazmente y a su vez prolongaron el poder correctivo por más de 61 días, condición que le permite ser una alternativa para solucionar problemas de acidez en el suelo.

\section{AGRADECIMIENTO}

A los Laboratorios de "Recursos Naturales" y "Suelos y Foliares" del Centro de Investigaciones Agronómicas de la Universidad de Costa Rica.

\section{LITERATURA CITADA}

Alcarde, JC. 1992. Corretivos da acidez dos solos: características e interpretacoes técnicas. ANDA, Sao Paulo, Brasil. Boletim Técnico No. 6. 26 p.

Alvarado, A; Fallas, J. 2004. La saturación de acidez y el encalado sobre crecimiento de la teca (Tectona grandis L.f) en suelos ácidos de Costa Rica. Agronomía Costarricense 28(1):81-87.

Alvarado, A; Molina, E; Cabalceta, G. 2010. Acidez y encalado de suelos. In Usón Murillo A. et al. (eds.). Tecnología de suelos: estudio de casos. Prensas Universitarias de Zaragoza, Ediciones de la Universidad de Lleida, Zaragoza, España. p. 69-99.

Andric, L; Rastija, M; Teklic, T; Kovacevic, V. 2012. Response of maize and soybeans to liming. TUBITAK. Turk J Agric. 36(1):415-420.

Azevedo, L; Avanzi, J; Carvalho, R; Silva, F; Rezende, J; Resende, A; Barreto, G. 2011. Nutrição e produção de matéria seca de milho submetido a calagem e adubação sulfatada. Pesq. Agropec. Trop. Goiânia 41(2):193-199.

Barber, S. 1984. Liming materials and practices. In Adams, F. (ed.). Soil Acidity and Liming. ASA, Wisconsin. p. 171-209.

Berettino, D; Loredo, J. 2005. Acidificación de suelos y aguas: problemas y soluciones. 7 ed. Instituto Geológico y Minero de España. Madrid, España. 212 p. 
Bertsch, F. 1995. La fertilidad de los suelos y su manejo. Asociación Costarricense de la Ciencia del Suelo. San José, Costa Rica. 157 p.

Braeuner, M; Ortiz, R; Macvean, C. 2005. Efectos de la aplicación de cal dolomítica y yeso agrícola en cafetales (Coffea arabica) afectados con mal de viñas en Guatemala. Manejo Integrado de Plagas y Agroecología 76(1):17-24.

Caires, E; Barth, E; Garbuio, F; Kusman, M. 2002. Correção da acidez do solo, crescimento radicular e nutrição do milho de acordo com a calagem na superfície em sistema plantio direto. Rev. Bras. Cien. Solo. 26(1):1011-1022.

Caires, E; Garbuio, F; Churka, S; Barth, G; Correa, J. 2008. Effects of soil acidity amelioration by surface liming on no-till corn, soybean, and wheat root growth and yield. European Journal of Agronomy 28(1):57-84.

Camacho, M; Cabalceta, G; Molina E. 2015. Efecto de las enmiendas líquidas en un Ultisol cultivado con sorgo. Agronomía Mesoamericana 26(2):291-303.

Clarkson, D; Hanson, J. 1980. The mineral nutrition of higher plants. Ann. Rev. Plant Physiol. 31:239-298.

Díaz-Romeu, R; Hunter, A. 1978. Metodología de muestreo de suelos, análisis químico de suelos y tejido vegetal e investigación en invernadero. Turrialba, Costa Rica, CATIE. 68 p.

Echeverría, H; Sainz, H; Calviño, P; Barbieri, P. 2001. Respuesta del cultivo de trigo al encalado. Instituto de Innovación y Transferencia de Tecnológica Agropecuaria (en línea). Buenos Aires, Argentina. Consultado 01 ago. 2013. Disponible en http:// agrolluvia.com/wp-content/uploads/2009/09/_b_respuesta-del-cultivo-de-trigo-al-encalado.pdf

Elizondo, J; Boschini, C. 2001. Efecto de la densidad de siembra sobre el rendimiento y calidad de forraje de maíz. Agronomía Mesoamericana 12(2):181-187.

Espinosa, J. 1995. Acidez y encalado de los suelos. Informaciones Agronómicas (INPOFOS) 20(1):6-14.

Espinosa, J; Molina, E. 1999. Acidez y encalado de los suelos. Quito, Ecuador, International Plan Nutrition Institute. $42 \mathrm{p}$.

Flores, E. 1999. Geografía de Costa Rica. San José, Costa Rica, Universidad Estatal a Distancia. 372 p.

Furtini, AE; Vilela, A; Ribeiro, F; Ribeiro, I. 1999. Liming effects on growth of native woody species from brazilian savannah. Pesq. Agropec. Bras. 34(5):829-837.

Gómez, T; Augusto, M; Espitia, U; Eder, J. 1996. Evaluación del comportamiento agronómico, crecimiento y desarrollo de ocho genotipos de maíz (Zea mays L) bajo estrés de acidez por aluminio en los suelos de Ayapel (Córdoba). Córdoba, España, Universidad de Córdoba. 70 p.

Henríquez, C; Cabalceta, G. 2012. Guía práctica para el estudio introductorio de los suelos con un enfoque agrícola. 2 ed. Asociación Costarricense de la Ciencia del Suelo. San José, Costa Rica. 111 p.

IICA (Instituto Interamericano de Cooperación Agrícola, Costa Rica). 1988. Manual de métodos analíticos de formulaciones de plaguicidas. San José, Costa Rica, IICA. 192 p.

López, I. 1986. Efecto de diferentes fuentes de enmienda sobre la reacción del suelo y respuesta del cultivo de sorgo (Sorghum vulgare pers). Agronomía Tropical 36(1):129-141

Lynch, J; Van Been, J. 1993. Growth and architecture of seedling roots of common bean genotypes. Crop Sciences 33(1):1253-1257.

Manetti, F; Fernandes, D. 2008. Momento de aplicação de calcário e gesso em um latossolo vermelho distrófico, no desenvolvimento inicial do milho. Rev. Botucatu (Brasil) 23(2):14-30.

Marschner, P; Marschner, H. 2012. Marschner's Mineral Nutrition of Higher Plants. 3rd ed. Londres, Inglaterra, Elsevier/Academic Press. 651 p.

Mills, H; Jones, JB. 1996. Plant analysis handbook II A practical sampling, preparation, analysis and interpretation guide. Georgia, USA, Micromacro Publish. p. 191.

Molina, E. 1998. Encalado para la corrección de la acidez del suelo. San José, Costa Rica, Asociación Costarricense de la Ciencia del Suelo. 45 p.

Molina, E; Rojas, A. 2005. Efecto del encalado en el cultivo de naranja valencia en la Zona Norte de Costa Rica. Agronomía Costarricense 29(3):81-95.

Muena, V; González, I; Neaman, A. 2010. Efectos del encalado y la fertilización nitrogenada sobre el desarrollo de Oenothera affinis en un suelo afectado por la minería del cobre. Rev. Cien. Suelo Nutr. Veg. 10(2):102-114.

Peters, JB; Kelling, KA; Schulte, EE. 1996. Choosing between liming materials. Madison, USA, University of Wisconsin Extension Cooperative Publication A3671. 4 p.

Pire, R. 1985. Densidad longitudinal de raíces y extracción de humedad en un viñedo del Tocuyo Venezuela. Agronomía Tropical 35(1/3):5-20.

Quero, E. 2005. El pH y su importancia en el manejo de la producción agrícola. Urupan, México, Instituto Tecnológico Superior de Urupan. 4 p.

Salager, J. 1992. El mundo de los surfactantes. Universidad de los Andes. Facultad de Ingeniería Química. Mérida, Venezuela. Consultado 09 jul. 2013. Disponible en http://www.firp.ula.ve/archivos/ cuadernos/S311A.pdf

Salas, R; Molina, E; Bouldin, D. 1996. Lime response on tanier in an Ultisol of Costa Rica. Communications in Soil Science and Plant Analysis 27(9/10): 2477-2484.

Sanchez, F. 2006. Características de los formulados comerciales de plaguicidas. Influencia sobre los 
residuos encontrados en alimentos (en línea). Almería, España. Consultado 01 ago. 2013. Disponible en http://dialnet.unirioja.es/servlet/ articulo?codigo $=1039289$

Solórzano, P. 1974. Efecto de la aplicación de dolomita en el crecimiento de maíz y soya, y cambios diferenciales en el $\mathrm{pH}$ de la rizosfera de estas especies como posible mecanismo de tolerancia a condiciones de acidez del suelo. Congreso Venezolano de Ciencias de Suelo (3, 1974, Maracay, Venezuela). Maracay, Venezuela. 2 p.

Soratto, P; Crusciol, C. 2008. Dolomite and phosphogypsum surface application effects on annual crops nutrition and yield. Agronomy Journal 100:261-270.
Tissi, J; Fávero, E; Pauletti, V. 2004. Efeitos da calagem em semedura direta de milho. Bragantia 63(3):405-413.

Valerio, J; Molina, E. 2012. Evaluación de una fuente de enmienda líquida en el rendimiento del arroz en un Ultisol de la zona norte de Costa Rica. Agronomía Costarricense 36(1):89-96.

Villalobos, E. 2001. Fisiología de la producción de los cultivos tropicales. Procesos fisiológicos básicos. San José, Costa Rica, Universidad de Costa Rica. 228 p.

Zapata, R. 2004. La química de la acidez del suelo. Medellín, Colombia, Sociedad Colombiana de la Ciencia del Suelo. 207 p. 